\title{
REDUCCIÓN DEL NIVEL DE POTASIO EN VINAZA DE DESTILERÍA UTILIZANDO RESINAS DE INTERCAMBIO IÓNICO
}

\author{
REDUCTION OF THE POTASSIUM LEVEL IN THE DISTILLATION \\ VINASSE USING ION EXCHANGE RESIN
}

\author{
Luisa Fernanda Torres Gaviria ${ }^{1}$, Juan Camilo Ocampo Vélez ${ }^{2}$, \\ Alcira Socarrás Cárdenas ${ }^{3}$ \\ 1,2 Química Industrial, Universidad Tecnológica de Pereira, Colombia \\ ${ }^{3}$ Ing. Msc Química, Universidad Tecnológica de Pereira, Colombia

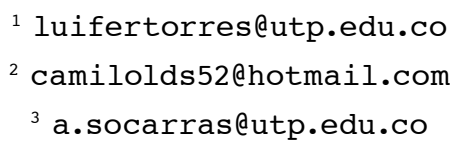

\section{RESUMEN}

En la producción de azúcar a partir de caña de azúcar se genera un subproducto denominado melaza, el cual es utilizado para la elaboración de alcohol carburante y este a su vez genera un nuevo residuo denominado vinaza. En promedio, se generan de 10 a 15 litros de vinaza por cada litro de alcohol producido. La vinaza tiene un alto contenido de materia orgánica y de micronutrientes que si no se manejan adecuadamente pueden contaminar el medio ambiente. Dentro de estos minerales se encuentra el potasio entre 10 y $18 \mathrm{mg} / \mathrm{ml}$ aproximadamente, el cual limita la utilización de la vinaza como fertilizante debido a que puede contaminar fuentes de agua y suelo con potasio y esto a su vez, restringe su utilización como componente en las formulaciones para concentrados de animales. Por tal razón, en el presente trabajo se realizó la disminución de potasio en vinazas provenientes de destilería de un ingenio azucarero de la región utilizando resina de intercambio catiónica fuerte y luego se utilizó una solución de ácido sulfúrico $4 \%$ como eluente para regenerar la resina de intercambio. Se obtuvo una disminución entre $97-98 \%$ del contenido inicial del potasio en la vinaza y una recuperación de potasio (99\%) como una solución de sulfato de potasio, la cual puede ser utilizada nuevamente como fertilizante líquido. La vinaza decationizada podría utilizarse como fertilizante orgánico en mayor proporción en los cultivos de caña de azúcar.

Palabras clave: Decationización; fertilizante; intercambio iónico; potasio; separación; vinaza.

\section{ABSTRACT}

In the production of sugar from sugarcane, a byproduct called molasses is generated, which is used in the production of fuel alcohol this 
in turn generates a new residue called vinasse. On average, 10 to 15 liters of vinasse are generated by liter of alcohol produced. Vinasse has a high content of organic matter and micronutrients, which pollute the environment because of an inadequate handled. Within these minerals is potassium between 10 and $18 \mathrm{mg} / \mathrm{ml}$, approximately, which limits the use of vinasse as a fertilizer due to the contamination of water sources and soil with this metal and restring its use as component in the formulations of animal food. In consequence, this paper presents the results of the reduction of potassium in distillation vinasses from a sugarmill from the region by a strong cation exchange resin and then, a sulphuric acid solution (4\%) was used as eluent for regenerate the exchange resin. A reduction between 97 - $98 \%$ was obtained from the initial contain of potassium in the vinase and potassium recovery was $99 \%$ as a potassium sulphate solution, which can be used again as liquid fertilizer. Decationized vinasse could be used as organic fertilizer in higher proportion in the sugarcane crops.

Key words: Decationization, fertilizer, ion exchange, potassium, vinasse.

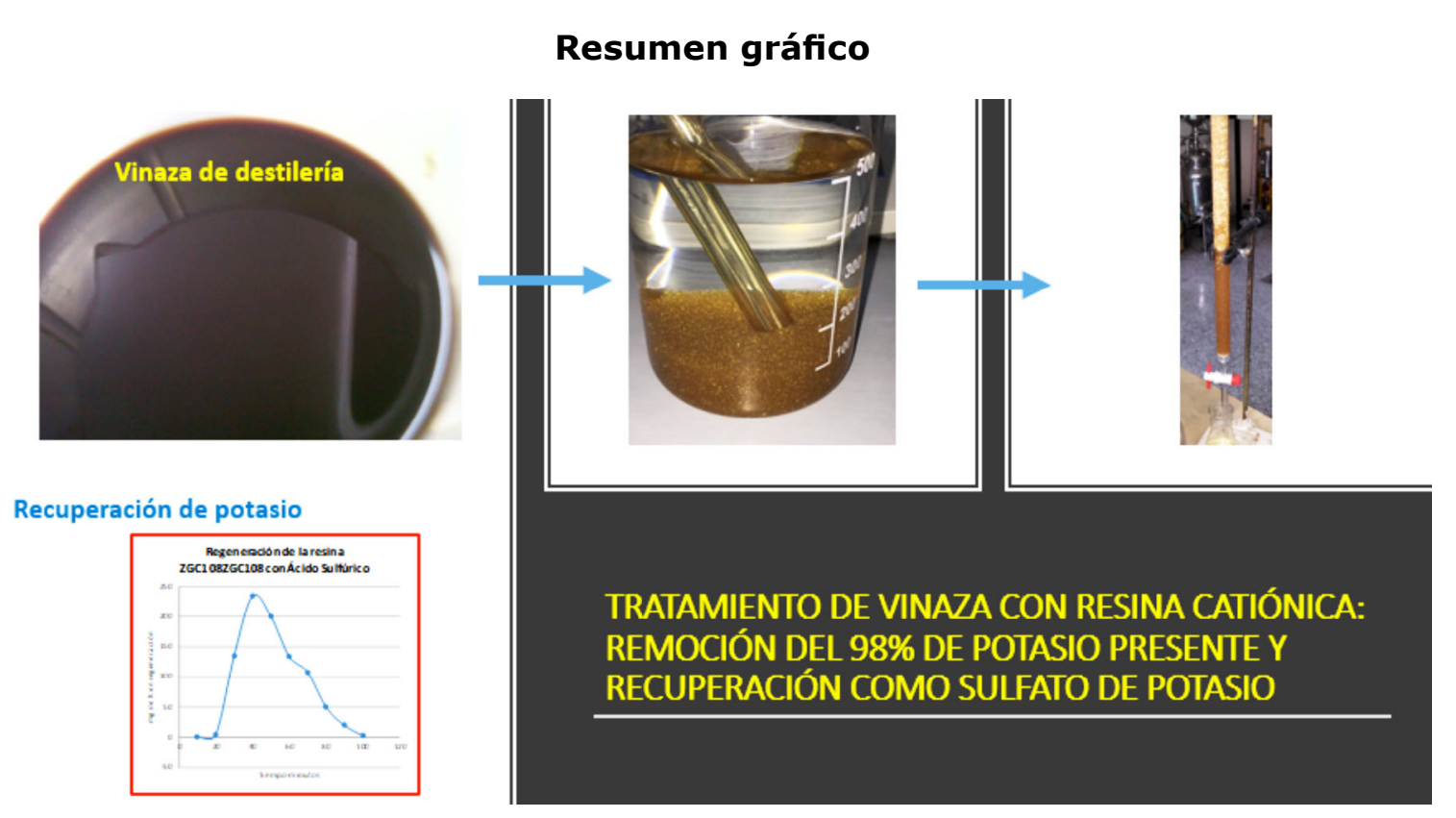

\section{INTRODUCCIÓN}

Las vinazas son el residuo líquido generado por las industrias licoreras durante el proceso de destilación del mosto fermentado para la obtención de alcohol, como el brandy, el ron, la cachaza y el bioetanol (Cerón, 2013). Este residuo o subproducto, se caracteriza por ser un líquido con un gran contenido de sólidos suspendidos, de color marrón o café oscuro, sabor a malta y olor a miel. En promedio se generan de 10 a 15 litros de vinaza por cada litro de alcohol producido, dependiendo de los equipos disponibles en la destilería. Su alto potencial contaminante es de aproximadamente 100 veces mayor que la de las aguas residuales domésticas, principalmente debido a su pH tan bajo ( $\mathrm{pH}: 3.5-5)$. Además, de altas concentraciones de potasio y sulfatos (García, 2006). 
La vinaza está compuesta por materiales orgánicos y nutrientes minerales que hacen parte de compuestos y constituyentes vegetales como aminoácidos, proteínas, lípidos, ácidos diversos, enzimas, bases, ácidos nucleicos, clorofila, lignina, quinonas, ceras, azúcares entre otras. Un elemento abundante en su composición es el potasio; su origen en la vinaza proviene directamente de la planta de caña de azúcar cuando se produce bioetanol a partir de esta materia prima (Lezcano, 2010).

Debido a su riqueza en minerales algunos ensayos como enmiendas al suelo y fertilizantes en el cultivo de la caña de azúcar han sido realizados en diferentes países como Cuba, Colombia y Brasil; a pesar de obtenerse mejoras en sus rendimientos su utilización ha está limitada al tipo de suelo, bajo pH (Aristizabal, 2015). El bajo contenido de sólidos totales (entre 8 y 10\%) también reduce su utilización cuando se trata de transportarlo. Se encuentran ensayos con resultados promisorios como la digestión anaerobia en plantas para producir biogás y que puede utilizarse como fuente de energía en calderas, con un ahorro significativo del combustible convencional. El lodo residual puede emplearse como alimento animal o como biofertilizante (Zhang, 2012). La vinaza puede mezclarse con otros elementos para elaborar concentrados para animales aportando un contenido de proteína cercano al $5.68 \%$, sales minerales y energía, sustituyendo parte de la melaza usada en la suplementación de ganado bovino, porcino y conejos, sin embargo, en bovinos dosis de potasio superiores a 1,5 Kg/animal causan efectos laxantes, razón por la cual es restringida su utilización (Gómez, 2000). La vinaza posee un gran valor como fertilizante, su alto contenido de materia orgánica y de micronutrientes, permite que se reutilice en fertirrigación en los cultivos de caña de azúcar. Sin embargo, cuando se utiliza en grandes cantidades, la vinaza está relacionada con la salinización del suelo y la contaminación de aguas superficiales y subterráneas. Es por esta razón, que la presente investigación tiene como propósito disminuir el nivel de potasio en vinaza proveniente de destilería por medio de una resina de intercambio iónico; para que de esta manera se pueda aprovechar en mayor cantidad como fertilizante o en alimentación animal (Marulanda, 2004).

Las resinas de intercambio iónico están constituidas por una matriz polimérica de elevado peso molecular, son insolubles, contienen grupos funcionales (positivos o negativos) capaces de intercambiar iones con una solución, estos grupos funcionales de las resinas intercambian en el caso más sencillo iones $\mathrm{H}+\mathrm{y}$ $\mathrm{OH}-$, presentes en la solución según sea el tipo de intercambiadores (Quintero, 2004).

Las resinas de intercambio iónico se pueden clasificar como:

- Resinas catiónicas de ácido fuerte: eliminan los cationes intercambiando sodios o protones.

- Resinas catiónicas de ácido débil: eliminan los cationes asociados con bicarbonatos.

- Resinas aniónicas de base fuerte: eliminan todos los aniones. Se utilizan para eliminar carbonatos y silicatos.

- Resinas aniónicas de base débil: eliminan con gran eficiencia los aniones de ácidos fuertes (sulfatos, nitratos y cloruros). 


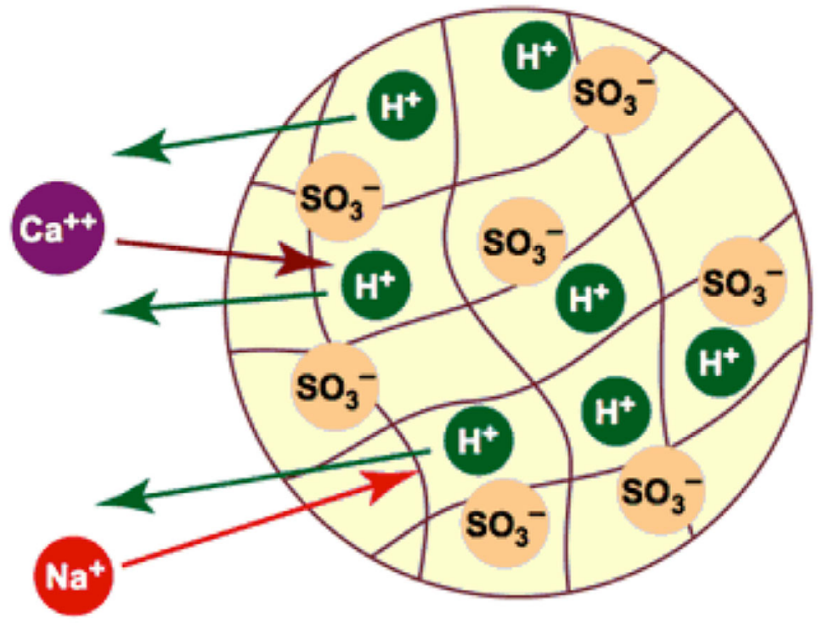

Figura 1. Decationización (todos los cationes reemplazados por $\left.\mathrm{H}^{+}\right)$.

Fuente: Dardel, 2017.

Las resinas catiónicas fuertes son capaces de eliminar todos los cationes de una solución. Presentan máxima selectividad para los cationes trivalentes, intermedia para los bivalentes e inferior para los monovalentes. La velocidad de intercambio es rápida y con poca fuga iónica. Son resinas muy estables y pueden durar hasta 20 años o más. Se hinchan poco, menos del $8 \%$, al pasar de la forma $\mathrm{Na}^{+}$a la $\mathrm{H}^{+}$. Las resinas catiónicas soportan temperaturas altas de más $100{ }^{\circ} \mathrm{C}$. Una resina de intercambio iónico, cuando se le ha agotado la capacidad de intercambiar iones, mediante una solución regenerante, pueden recuperar completamente su capacidad de intercambio original. La regeneración de la columna se consigue haciendo pasar a través de la resina una solución con el ion original, el cual se une a los radicales de la resina y desplaza a los iones captados durante el funcionamiento normal (Perry, 2001).

\section{MATERIALES Y REACTIVOS}

- Resina de intercambio catiónica ZGC108 (Hangzhou Zheng Guang Co., Ltd, China) la cual cuenta con un grupo sulfónico $\left(-\mathrm{SO}_{3} \mathrm{H}\right)$ en el copolímero de estirenodivinilbenceno y se encuentra en forma sódica $\mathrm{Na}^{+}$, presenta buena capacidad de intercambio (>1000 mmol/L), estabilidad física y trabaja en un rango de $\mathrm{pH}$ de 1-14.

- Columnas de vidrio de $35 \mathrm{~cm}$ de longitud y $1 \mathrm{~cm}$ de diámetro con filtro, por las cuales se colocó en contacto la resina y la vinaza para llevar a cabo el intercambio.

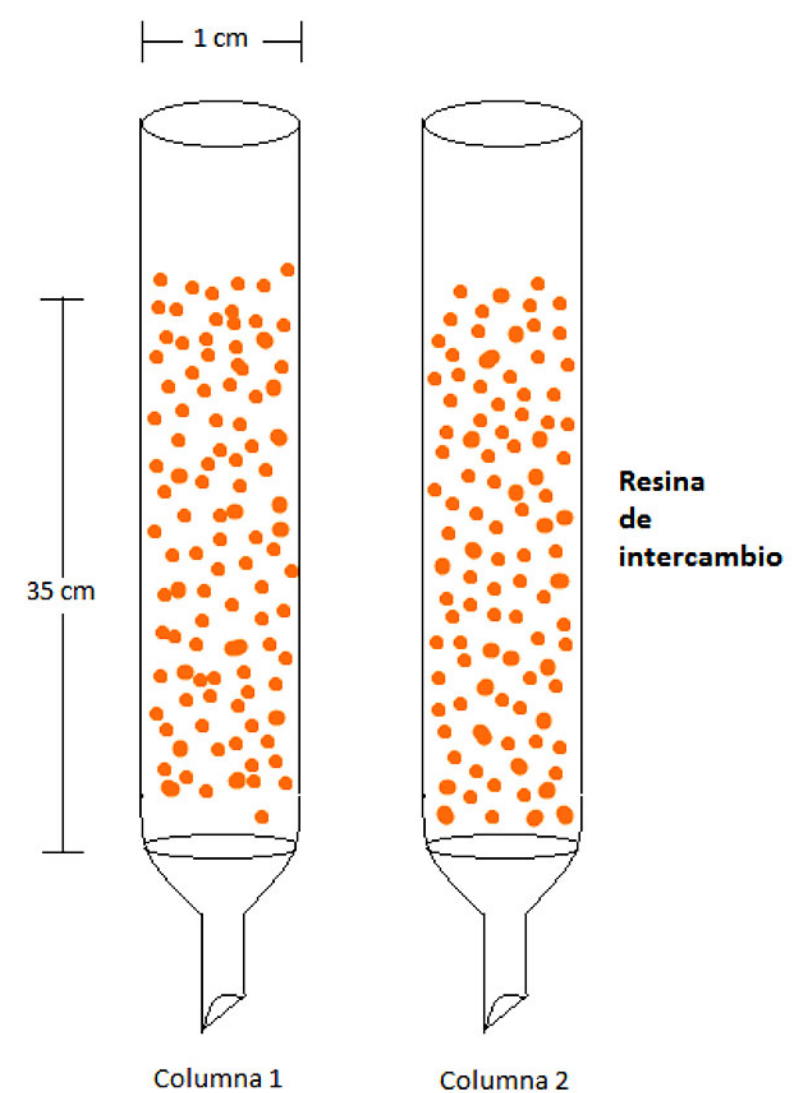

Figura 2. Diseño de columnas utilizadas para el intercambio. Fuente: Autores.

- Bomba peristáltica, utilizada para manejar el flujo requerido en el intercambio y la regeneración de la resina.

- Equipo de absorción atómica: SHIMADZU AA-7000. No. de serie A306647 para realizar la determinación de potasio y otros cationes

- Ácido Sulfúrico y $\mathrm{NaOH}$ grado reactivo marca Sigma. 
- Baño termostático para mantener la temperatura constante.

\section{METODOLOGÍA}

La muestra de vinaza para llevar a cabo el desarrollo de la presente investigación es obtenida directamente de la sección de destilería de un ingenio azucarero de la región. Presenta un color marrón con una gran cantidad de sólidos suspendidos y decantados, es completamente líquida y tiene un olor fuerte a miel.

\section{Caracterización de vinaza}

La composición de la vinaza depende de las características de la materia prima utilizada en la producción de alcohol, ya sea de melaza, de los jugos de caña, de los nutrientes utilizados en la fermentación, de la eficiencia del proceso y de la maduración de la caña (Betancourt, 2012).

El desarrollo experimental de esta investigación se realizó con muestras obtenidas directamente de una destilería de la región. Las muestras se mantuvieron bajo refrigeración a $4^{\circ} \mathrm{C}$ y se realizó la determinación de algunas propiedades importantes para su caracterización, como densidad, grados brix, acidez, pH, sólidos totales y concentración de potasio y los resultados se presentan en la Tabla 1 (Leal, 2003).

La determinación de $\mathrm{pH}$ se realizó potenciométricamente de acuerdo con Standard Methods for the Examination of Water and Wastewater 23 RD Edition No. 4500. Para los sólidos totales se utilizó la técnica de gravimetría con secado hasta peso constante y se determinó el contenido de humedad mediante el método APHA 2540 B; la densidad se calculó utilizando un picnómetro; la acidez se estimó por titulación utilizando $\mathrm{NaOH}$ como agente para neutralización. La cuantificación de potasio, magnesio, hierro, zinc, cobre, calcio y sodio se realizó por espectrometría de absorción atómica con llama con un espectrofotómetro SHIMADZU A.A.
7000. Para la determinación de calcio por este método se utilizó una lámpara de cátodo hueco para calcio y la mezcla de gases óxido nitrosos-acetileno alcanzándose temperatura aproximada entre 2900 y $3000^{\circ} \mathrm{C}$. Para los demás metales también se utilizó lámpara de cátodo hueco dependiendo del metal y para todos los casos una mezcla de gas acetileno-aire.

\section{Pretratamiento de la resina}

La resina ZGC108 se encontraba en forma sódica y se cambió a forma ácida (recomendación del fabricante) para que esta pudiera retener los iones de potasio disueltos en la vinaza. Se le adicionó a la resina una cantidad de agua destilada equivalente a tres veces su volumen, dejándola en reposo por 24 horas, posteriormente se filtró y se trató con una solución de $\mathrm{NaOH} \mathrm{0,1} \mathrm{M.} \mathrm{Finalmente,} \mathrm{se} \mathrm{trató}$ con una solución de $\mathrm{HCl}$ 0,1 M esperando entre cada adición 6 horas, para así hacer la respectiva medición de $\mathrm{pH}$; obteniendo un valor de aproximadamente 6,5 . Se continuó con los siguientes ensayos con la resina empacada en la columna.

\section{Reducción del contenido de potasio en vinaza utilizando resina de intercambio iónico}

Para llevar a cabo la reducción se utilizaron dos columnas de vidrio de $35 \mathrm{~cm}$ de longitud y $1 \mathrm{~cm}$ de diámetro cada una, en las cuales se realizó la interacción entre la vinaza y la resina. En cada columna se colocaron 220 gramos para garantizar la eliminación de cationes, específicamente de potasio.

La resina en evaluación tiene una capacidad de intercambio de 2000 equivalentes/L y la vinaza que se utilizó tenía un contenido de 671,2 equivalentes/L por lo cual se tomaron $120 \mathrm{~mL}$ de vinaza y se pasaron por las dos columnas con un flujo de $2 \mathrm{~mL} / \mathrm{min}$ durante dos horas, tomando muestras cada 30 minutos. 
En esta parte del proceso se realizaron dos ensayos, uno con vinaza a temperatura de $28^{\circ} \mathrm{C}$ y otro con vinaza a $40^{\circ} \mathrm{C}$. Los mejores resultados se obtuvieron con la vinaza a temperatura de $40^{\circ} \mathrm{C}$, por tal razón los ensayos dos y tres se trabajaron a esta temperatura, además, fue posible reducir el tiempo de intercambio a una hora.

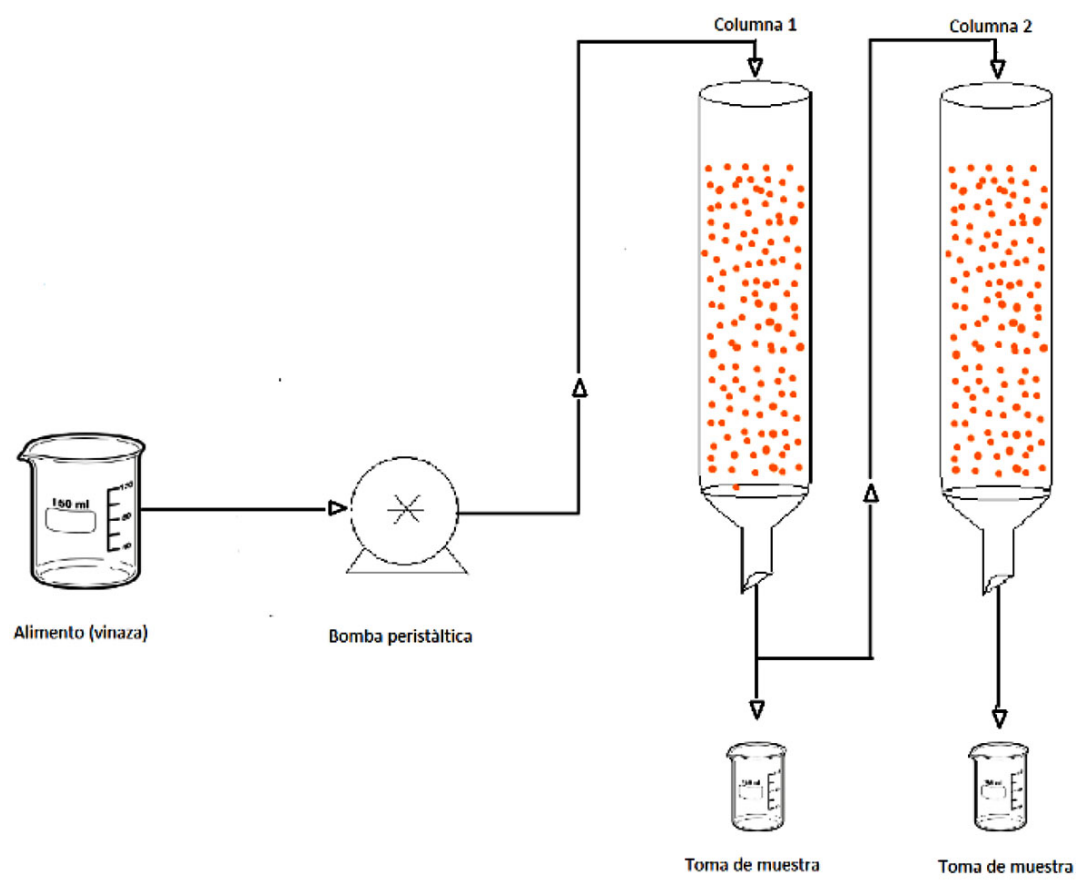

Figura 3. Proceso de intercambio utilizado en laboratorio.

Fuente: propia.

Regeneración de la resina de intercambio Se realizó la regeneración de la resina saturada anteriormente con los cationes con el propósito de liberar el potasio y cargarla nuevamente con $\mathrm{H}^{+}$

Se utilizó ácido sulfúrico (recomendación del fabricante) como agente regenerante a diferentes concentraciones $(0,2 \mathrm{M}, 0,3 \mathrm{M}, 0,4 \mathrm{M}$, $0,5 \mathrm{M}$ y $0,6 \mathrm{M})$ para encontrar la concentración adecuada para el proceso de regeneración. Se tomó 5 veces el volumen del ácido con respecto al volumen de resina. Para el primer ensayo se colocaron ambos (resina y ácido) en un beaker con agitación constante a $35^{\circ} \mathrm{C}$ y se tomaron muestras cada $20 \mathrm{mi}-$ nutos durante 3 horas, finalmente se analizó el contenido de potasio por espectroscopía de absorción atómica. Para los ensayos dos y tres la regeneración de la resina se llevó a cabo en las columnas, manejando un flujo de $2 \mathrm{~mL} / \mathrm{min}$, bajo las mismas condiciones de temperatura, y se redujo el tiempo de contacto entre el regenerante y la resina a 100 minutos (Dardel, 2016).

\section{Obtención de cristales de sulfato de po- tasio}

Las muestras obtenidas después de la regeneración de la resina fueron sometidas a un proceso de cristalización con el propósito de recuperar el potasio como sulfato de potasio en solución. En la figura 4 se explica el procedimiento utilizado. 


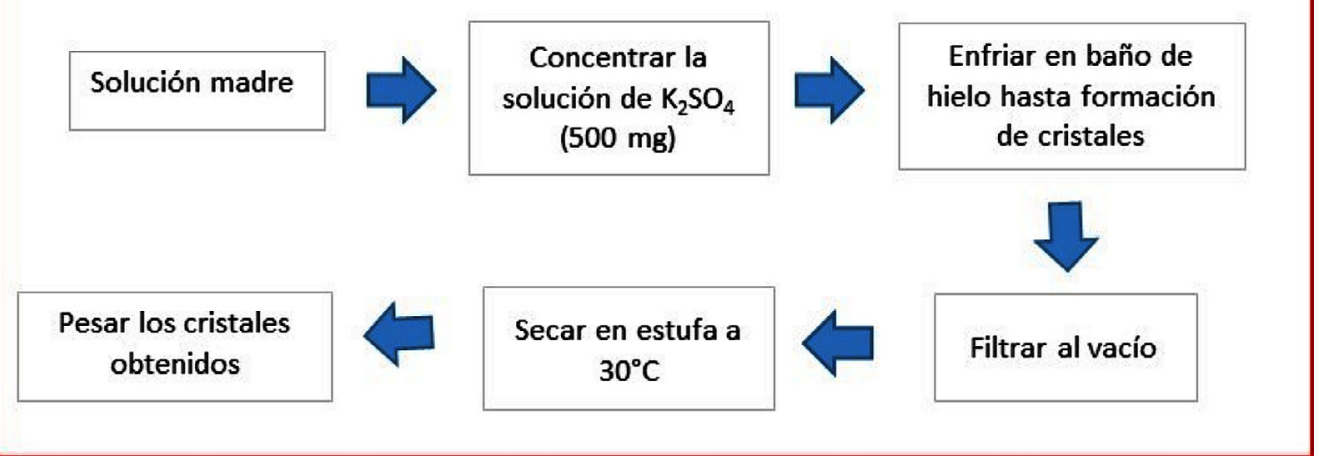

Figura 4. Procedimiento obtención de cristales.

\section{RESULTADOS Y DISCUSIÓN}

\section{Caracterización vinaza}

La vinaza proveniente de la destilería fue caracterizada, en la Tabla 1 se muestran los resultados obtenidos, los cationes se analizaron por absorción atómica. Se puede observar que el contenido de potasio es de 9,32 mg/ml siendo este el metal de mayor concentración presente en la vinaza y se verifica que la vinaza es ácida con un pH de 4,3.

Tabla 1. Resultados caracterización vinaza.

\begin{tabular}{cc}
\hline PRUEBA & Resultado \\
\hline GRADOS BRIX & 14,03 \\
DENSIDAD $(\mathrm{g} / \mathrm{mL})$ & 1,09 \\
& \\
POTASIO $(\mathrm{mg} / \mathrm{mL})$ & 9,32 \\
MAGNESIO $(\mathrm{mg} / \mathrm{mL})$ & 2,22 \\
HIERRO $(\mathrm{mg} / \mathrm{mL})$ & 0,83 \\
ZINC $(\mathrm{mg} / \mathrm{mL})$ & 0,07 \\
COBRE $(\mathrm{mg} / \mathrm{mL})$ & 0,03 \\
CALCIO $(\mathrm{mg} / \mathrm{mL})$ & 2,42 \\
SODIO $(\mathrm{mg} / \mathrm{mL})$ & 1,27 \\
SÓLIDOS TOTALES $(\mathrm{g} / \mathrm{mL})$ & 1,00 \\
pH & 4,30 \\
\hline
\end{tabular}

\section{Reducción del contenido de potasio en vinaza utilizando resina de intercambio iónico}

Las resinas de intercambio tienen preferencia por ciertos iones que poseen unas características de carga similares. La carga y el tamaño de los iones juegan un papel muy importante y tienen influencia sobre la selectividad, debido a que una resina prefiere contraiones de elevada valencia. El orden de selectividad para los metales alcalinos es:

$\mathrm{Cs}+>\mathrm{Rb}+>\mathrm{K}+>\mathrm{Na}+>\mathrm{Li}+$ y para los alcalinotérreos: $\mathrm{Ba} 2+>\mathrm{Sr} 2+>\mathrm{Ca} 2+>\mathrm{Mg} 2+>\mathrm{Be} 2+$.

Inicialmente se llevaron a cabo dos ensayos por triplicado con el objetivo de observar la temperatura adecuada en el proceso de intercambio. Se evaluaron temperaturas de $28^{\circ} \mathrm{C}$ y $40^{\circ} \mathrm{C}$. Ambos ensayos se realizaron bajo las mismas condiciones de tiempo y flujo, sin embargo, como se puede observar los datos promedio en la Tabla 2, se obtuvieron mejores resultados con temperatura de $40^{\circ} \mathrm{C}$, logrando una remoción de potasio del 0,52 \% más con respecto a la temperatura de $28^{\circ} \mathrm{C}$.

Para los ensayos 3 y 4 de la Tabla 2, se utilizó temperatura de $40^{\circ} \mathrm{C}$ y se redujo el tiempo de 
intercambio a 1 hora, con el mismo flujo y utilizando una solo columna para cada ensayo, se obtuvieron porcentajes de remoción por encima del 97,28 \% en promedio.

Estos resultados demuestran que la capacidad de intercambio de la resina depende de la temperatura, del tiempo y del flujo, este último es de gran importancia ya que se debe determinar la cantidad de vinaza que se debe pasar por unidad de tiempo para que la resina se sature e intercambie la mayor cantidad de potasio posible, permitiendo que la vinaza reduzca su contenido y aumente sus usos industriales teniendo en cuenta los equivalentes de la resina, que es la capacidad que tiene de retener iones (Becerra, 2014).

Tabla 2. Resultados reducción de potasio en vinaza.

\begin{tabular}{ccc}
\hline ENSAYO & \multicolumn{2}{c}{ Temperatura $\left({ }^{\circ} \mathrm{C}\right)$} \\
& 28 & 40 \\
\hline CONTENIDO INICIAL DE POTASIO EN & 457,92 & 457,92 \\
VINAZA (mg) & & \\
CONTENIDO FINAL DE POTASIO EN & 14,34 & 12 \\
VINAZA (mg) & 443,58 & 445,92 \\
TOTAL REMOCIÓN DE POTASIO (mg) & $44,77 \%$ & $98,03 \%$ \\
\% DE REMOCIÓN DE POTASIO & $97,77 \%$ & \\
& & \\
Temp 40 ${ }^{\circ} \mathrm{C}$ & Ensayo 2 & Ensayo 3 \\
\hline CONTENIDO INICIAL DE POTASIO EN & & \\
VINAZA (mg) & 918,75 & 918,75 \\
CONTENIDO FINAL DE POTASIO EN & & \\
VINAZA (mg) & 18,55 & 23,21 \\
TOTAL REMOCIÓN DE POTASIO (mg) & 900,19 & 895,53 \\
\% DE REMOCIÓN DE POTASIO & 0,9708 & 0,9747 \\
\hline
\end{tabular}

\section{Regeneración de la resina}

El objetivo de esta evaluación fue realizar la elución del potasio retenido en los sitios funcionales de la resina y para esto se evaluaron diferentes concentraciones de ácido sulfúrico, con el fin de determinar la adecuada para regenerar la resina.

Se utilizaron concentraciones de 0,2M, 0,3M, $0,4 \mathrm{M}, 0,5 \mathrm{M}$, y $0,6 \mathrm{M}$ por triplicado y los mejores resultados se obtuvieron con los ácidos 0,4 $M$ y $0,6 M$, logrando remover $82,44 \%$ y 82,65 $\%$ del potasio contenido en la resina, respectivamente (Tabla 3, valores promedio). Como los resultados de remoción fueron similares se decidió escoger la concentración más baja de ácido sulfúrico 0,4M.

Se realizaron tres ensayos por triplicado (uno en beaker y dos en columna) utilizando ácido sulfúrico $0.4 \mathrm{M}$ como regenerante, se pueden observar los valores promedio en la Tabla 4. Los mejores resultados se obtuvieron en los ensayos dos y tres, y teniendo en cuenta la variación que tuvieron estos con respecto al primer ensayo, se puede concluir que el proceso de regeneración de la resina resulta ser óptimo cuando se lleva a cabo en la columna y manejando flujos constantes, además, cabe resaltar que bajo las últimas condiciones mencionadas fue posible disminuir el tiempo 
de contacto entre el ácido y la resina a 100 minutos, obteniendo porcentajes de remoción de potasio mayores al $98 \%$.

Se utilizó ácido sulfúrico como regenerante debido a que es un ácido fuerte y tiene mayor capacidad de eliminar el potasio retenido por la resina, esto gracias a que los iones $\mathrm{H}^{+}$que contiene son liberados más fácilmente que un ácido débil, es decir, el ácido sulfúrico cede fácilmente sus protones.

Tabla 3. Evaluación de la concentración del ácido regenerante.

\begin{tabular}{cccccc}
\hline & \multicolumn{5}{c}{ CONCENTRACIÓN ÁCIDO SULFÚRICO (mol/L) } \\
& 0,2 & 0,3 & 0,4 & 0,5 & 0,60 \\
\hline TEMPERATURA ( $\left.{ }^{\circ} \mathrm{C}\right)$ & 35 & 35 & 35 & 35 & 35,00 \\
CONTENIDO INICIAL DE POTASIO EN & & & & & \\
RESINA (mg) & 441,24 & 441,24 & 441,24 & 441,24 & 441,24 \\
CONTENIDO FINAL DE POTASIO EN & & & & & \\
RESINA (mg) & 136,94 & 233,74 & 363,79 & 209,15 & 364,71 \\
TOTAL REMOCIÓN DE POTASIO (mg) & 304,3 & 207,5 & 77,45 & 232,09 & 76,53 \\
\% DE REMOCIÓN DE POTASIO & $31,04 \%$ & $52,97 \%$ & $82,44 \%$ & $47,40 \%$ & $82,65 \%$ \\
\hline
\end{tabular}

Tabla 4. Resultados regeneración resina con ácido 0,4M.

\begin{tabular}{cccc}
\hline & \multicolumn{3}{c}{ ENSAYO } \\
& 1 & 2 & 3 \\
\hline T $\left({ }^{\circ} \mathrm{C}\right)$ & 35 & 35 & 35 \\
CONTENIDO INICIAL DE POTASIO EN & & & \\
RESINA (mg) & 441,24 & 900,19 & 895,53 \\
CONTENIDO FINAL DE POTASIO EN & & & \\
RESINA (mg) & 78,85 & 11,31 & 4,74 \\
TOTAL REMOCIÓN DE POTASIO (mg) & 362,39 & 888,88 & 890,79 \\
\% DE REMOCIÓN DE POTASIO & $82 \%$ & $99 \%$ & $99 \%$ \\
\hline
\end{tabular}

En la figura 5 se puede observar la curva de regeneración de la resina en la columna. Durante los primeros 40 minutos se observa un comportamiento exponencial el cual nos indica la recuperación de este catión de potasio que está presente en la resina saturada en el eluente, después de este tiempo empieza una fase de reducción del contenido de potasio lo cual indica que el contenido de este metal en la resina está decayendo. Esto nos indica que la remoción se está realizando de manera adecuada bajo las condiciones de operación y se está liberando potasio como sulfato de potasio. 


\section{Regeneración de la resina ZGC108ZGC108 con Ácido Sulfúrico}

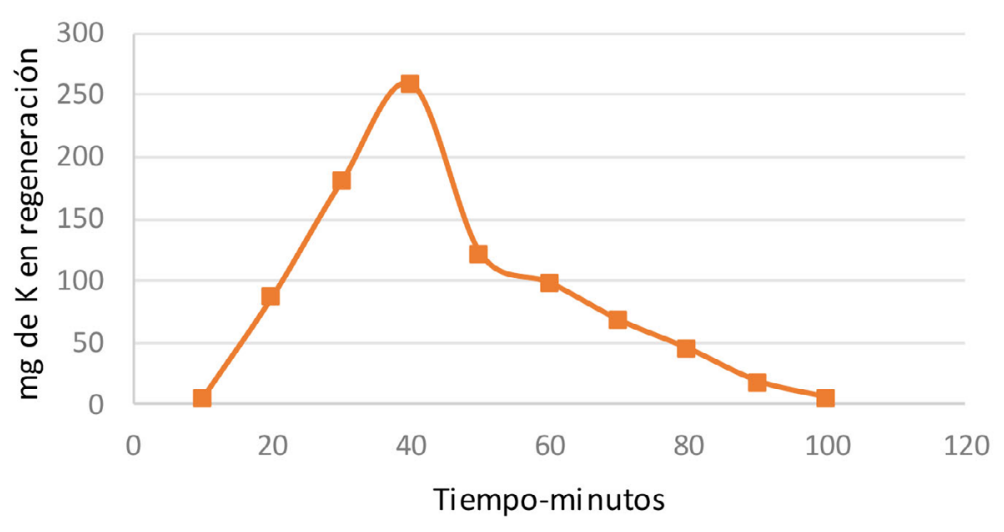

Figura 5. Regeneración: Disminución de potasio en resina con respecto al tiempo para el ensayo 1.

En la figura 6, los resultados son similares al primero, se observa que el punto más alto es en el minuto 40 donde se presenta la mayor liberación de potasio. En esta evaluación, el porcentaje de liberación de potasio tuvo un valor más alto que el primero eliminando $82,13 \%$ del contenido de potasio adherido en la resina. Se realizó una segunda determinación en donde se obtuvo un porcentaje de $98,74 \%$ de liberación de potasio contenido en la resina, se realizó un tercer ensayo para verificar la regeneración de la columna y se obtuvo un valor del 99,47\%. Esto indica que la resina está regenerada y sin la presencia de cationes por lo tanto se puede volver a utilizar en el proceso de intercambio catiónico.

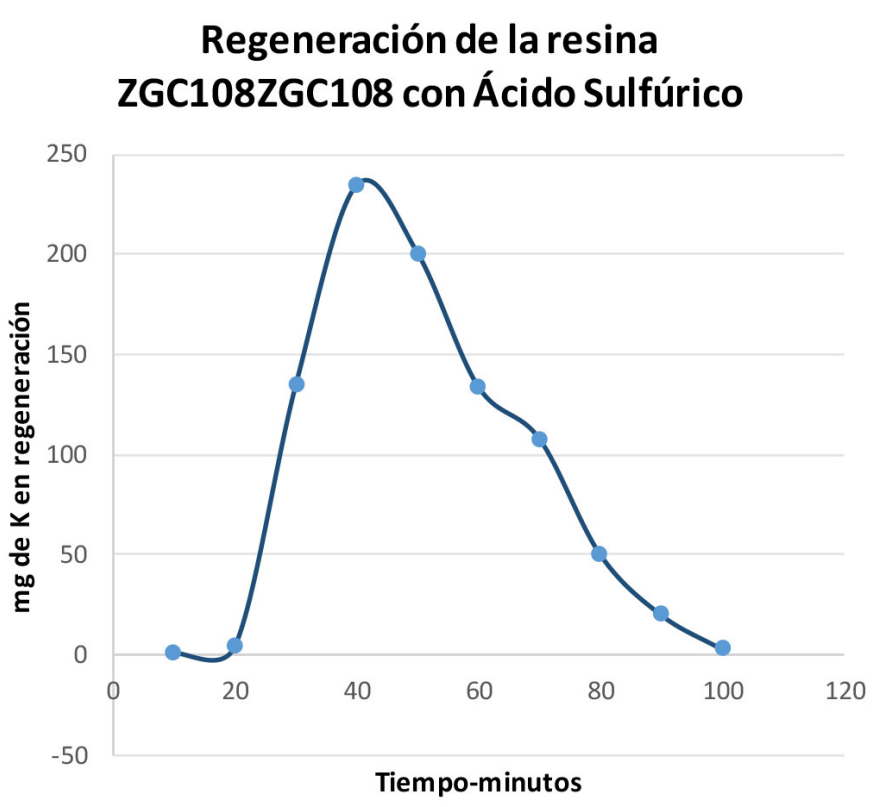

Figura 6. Disminución de potasio en resina con respecto al tiempo para el ensayo 2 . 


\section{Obtención de cristales de sulfato de potasio}

El proceso de obtención de cristales de sulfato de potasio se llevó a cabo a nivel de laboratorio utilizando tres eluentes obtenidos del proceso de regeneración. Primero se realizó una evaporación, posteriomente se procedió a una cristalización para lo cual se utilizó un balón con desprendimiento lateral conectado a una bomba de vacío, agitación en malla de calentamiento con temperatura de $70^{\circ} \mathrm{C}$. Luego se realizó un enfriamiento en un baño termostatado hasta bajar la temperatura a $20^{\circ} \mathrm{C}$ (Zhang, 2012)

Teniendo el $\mathrm{K}_{2} \mathrm{SO}_{4}$ en la solución, se procede a la recuperación de este en forma de cristales, el procedimiento se realiza en los tres ensayos, obteniendo sus respectivos porcentajes de rendimiento.

Tabla 5. Resultados de obtención de cristales.

\begin{tabular}{ccccc}
\hline & \multicolumn{3}{c}{ ENSAYO } \\
\hline PESO & $\begin{array}{l}\text { CRISTALES } \\
(\mathrm{mg})\end{array}$ & 0,003 & 0,0027 & 0,0033 \\
$\%$ RENDIMIENTO & 37 & 32 & 40
\end{tabular}

Fuente: Autores

En los resultados, el porcentaje de rendimiento es bajo, debido a que no se recupera todo en forma de cristal, quedando en la solución parte del sulfato de potasio como licor madre.

Los cristales obtenidos tienen uso comercial para la zona agroindustrial en forma de abono para la tierra. En nuestro estudio este cristal es almacenado para estudios posteriores.

\section{CONCLUSIONES}

Se logró un alto porcentaje de reducción en el nivel de potasio de la vinaza, el cual fue el objetivo principal de esta investigación, utilizando la resina ZGC108 en una relación de 0,36 Kg de resina / $L$ de vinaza. La cual presenta ventajas como el amplio rango de $\mathrm{pH}$ y las altas temperaturas que puede soportar durante el proceso de operación, demostrando que es efectiva para la reducción de iones de potasio de las vinazas de destilería removiendo el $98 \%$ del potasio alimentado al proceso.

El ácido sulfúrico presentó buenos resultados en la regeneración de la resina saturada, debido a que es un ácido fuerte y por lo tanto libera más fácilmente los iones $\mathrm{H}^{+}$lo cual permite que el ion sulfato pueda interactuar con el potasio presente en la resina liberando sulfato de potasio durante el proceso de regeneración.

Se recomienda optimizar el proceso de cristalización de sulfato de potasio; en caso de requerirse sólido para su utilización como fertilizante.

\section{LITERATURA CITADA}

Aristizábal, C. (2015) Caracterización fisicoquímica de una vinaza resultante de la producción de alcohol de una industria licorera, a partir del aprovechamiento de la caña de azúcar. Ing. USBMed, 6 (2).

Becerra, N. (2014). Clarificación de vinazas de caña de azúcar por tratamiento fisicoquímico y filtración con membranas (Tesis de maestría). Universidad Nacional de Colombia, Bogotá.

Betancourt, C; Quiñonez, W. (2012). Biodegradación anaeróbica de vinaza generada en la destilería de Soderal y aprovechamiento energético del biogás como medio para bajar el impacto de gases de efecto invernadero (Tesis de Maestría). Universidad Politécnica Salesiana, Ecuador.

Cerón, V. (2013). Caracterización Ambiental De Las vinazas de residuos De caña de azúcar Resultantes De La Producción De Etanol. Dyna, 177.

Dardel, F. (201-2018). El intercambio iónico. Lugar de publicación: http://dardel.info/IX/index_ ES.html

García, A.; Marulanda, E. y Puerto, O. (2004). Experiencias en el uso de vinazas en la agricultura vallecaucana CD Memorias Seminario. Vinazas, 
potasio y elementos menores para una agricultura sostenible. Sociedad Colombiana de la Ciencia del suelo.

Gomez E. (2000). Manual de los derivados de la industria azucarera. Instituto Cubano de Investigaciones de los Derivados de la Caña de Azúcar.

Leal, I. (2003). Caracterización fisicoquímica de la vinaza del Agave cocui y su posible uso agroindustrial. Multiciencias. Universidad de Zulia, 3(2).

Lezcano, P.; Mora, L. (2010). Las vinazas de destilería de alcohol. Contaminación ambiental o tratamiento para evitarlo. Instituto de Ciencia Animal, apdo.24. San José de las Lajas, La Habana.
Rojas, C. (2006). Posibilidades de uso de la vinaza en la agricultura de acuerdo con su modo de acción en los suelos. Tecnicaña.

Quintero, R. (2004). Perspectivas acerca del uso y manejo de vinazas aplicadas al suelo. CD Memorias seminario "Vinaza, Potasio y elementos menores para la agricultura sostenible. Sociedad Colombiana de las Ciencias del Suelo. Palmira.

Peerry, R. (2001). Manual del Ingeniero Químico. Mc Graw-Hill , 3. 7a edición.

Zhang, P. (2012). Using strong acid-cation exchange resin to reduce potassium level in molasses vinasses. Desalination 286 (1). 